\title{
Mammakarzinom: Neue Daten zum 10-Jahres-Überleben in Europa
}

Um die Prognose von Brustkrebspatientinnen besser beurteilen zu können, werden Daten zum Langzeitüberleben benötigt. Aktuelle europaweite Daten zum 10-Jahres-Überleben liegen vor.

m Rahmen der Eurocare-4-Studie wurden die Daten zum 10-Jahres-Überleben von 114.312 Frauen im Alter von 1599 Jahren überprüft, die in den Jahren 2000 bis 2002 erstmalig die Diagnose Brustkrebs erhielten. Datenquelle waren 24 bevölkerungsbasierte Krebsregister aus 14 europäischen Ländern. Von den Frauen, die zu irgendeinem Zeitpunkt in 2000 bis 2002 lebten, wurde im PeriodApproach-Verfahren das relative Überleben in den Jahren 1, 5 und 10 nach Diagnosestellung geschätzt. Außerdem wurde das 10-Jahres-Überleben in Abhängigkeit zum Überleben in Jahr 1 und 5 nach Diagnose geschätzt.

$49 \%$ der Frauen wurden in der Altersklasse 50-69 Jahre diagnostiziert, $31 \%$ in der Altersklasse 70-99 Jahre. Das
10-Jahres-Überleben überstieg in den meisten Regionen $70 \%$, die höchsten Werten registrierte man in Nordeuropa (etwa $75 \%$ ). In Osteuropa erreichten dagegen nur $54 \%$ der Frauen diese Überlebensmarke.

Das 10-Jahres-Überleben konditional zum Überleben in Jahr 1 lag in allen europäischen Ländern um 2-6\% höher als das 10-Jahres-Überleben. Das 10-JahresÜberleben der Frauen, die mindestens 5 Jahre überlebt hatten, betrug insgesamt $88 \%$ mit den höchsten Werten in UK (91\%) und den niedrigsten Werten in Osteuropa (79\%).

Frauen in der Altersgruppe 50-69 Jahre hatten ein höheres allgemeines Überleben als ältere und jüngere Frauen. In sechs Krebsregistern fanden sich ad- äquate Informationen zum Krankheitsstadium bei Diagnosestellung. Anhand dieser Daten wurde ein 10-Jahres-Überleben von $89 \%$ für lokal begrenzte Tumoren geschätzt, für lokoregionäre und metastasierte Erkrankungen betrugen die Raten 62 und $10 \%$.

Fazit: Das 10-Jahres-Gesamt-Überleben nach Brustkrebserstdiagnose liegt in den meisten europäischen Ländern bei über $70 \%$. Um noch aussagekräftigere Daten für einen Länder- und Altersvergleich zu erhalten, braucht man Angaben zum Krankheitsstadium bei Diagnosestellung. Diese Daten werden allerdings bisher kaum in die Register zum Mammakarzinom aufgenommen.

Barbara Kreutzkamp

Allemani $C$ et al. Predictions of survival up to 10 years after diagnosis for European women with breast cancer in 2000-2002. Int J Cancer. 2013;132(10):2404-12.

\section{Zweiter Angriffspunkt beim HER2-positiven Mammakarzinom}

\section{Trastuzumab gilt als etablierter Standard in der Behandlung des HER2- positiven, lokal fortgeschrittenen oder metastasierten Mammakarzinoms. Einen möglichen synergistischen antitumorellen Effekt versprach die Kombination mit Bevacizumab.}

$\mathrm{D}$ er gegen den epidermalen Wachstumsfaktorrezeptor HER2 gerichtete monoklonale Antikörper Trastuzumab verbessert signifikant das progressionsfreie Krankheitsintervall und Gesamtüberleben beim HER2-positiven, lokal fortgeschrittenen/metastasierten Mammakarzinom im Vergleich zur alleinigen Chemotherapie. Wie aus experimentellen und klinischen Daten hervorgeht, korreliert die HER2-Überexpression mit der Up-Regulation von VEGF (Vascular Endothelial Growth Factor) in malignen Zellen. Dieser Zusammenhang liefert die biologische Rationale für das parallele Targeting von HER2 plus VEGF bei Frauen mit einem HER2-positiven Mammakarzinom.

In der open-label, randomisierten und kontrollierten Phase-III-Studie AVEREL wurde der mögliche positive Effekt der Kombination von Trastuzumab mit dem Anti-VEGF-Antikörper Bevacizumab plus Docetaxel (BTH) gegenüber Trastuzumab/Docetaxel (TH) auf das progressionsfreie Übereben (PFS) beim lokal rezidivierten oder metastasierten HER2positiven Mammakarzinom geprüft. Eingeschlossen waren 424 geeignete Patientinnen in 16 Ländern ohne vorherige Trastuzumab- oder Chemotherapie. Die Patientinnen erhielten nach der Randomisierung entweder $100 \mathrm{mg} / \mathrm{m}^{2}$ Docetaxel plus eine Aufsättigungsdosis von $8 \mathrm{mg} / \mathrm{kg}$ Trastuzumab, gefolgt von $6 \mathrm{mg} /$ $\mathrm{kg}$ entweder mit (Verumarm) oder ohne (Vergleichsarm) $15 \mathrm{mg} / \mathrm{kg}$ Bevacizumab in dreiwöchigen Intervallen.

Nach einem medianen Follow-up von 26 Monaten betrug das PFS gemäß As- sessment der Autoren 13,7 Monate im Vergleichsarm vs. 16,5 Monate in der BTH-Gruppe. Die Ansprechraten erreichten $70 \%$ gegenüber $74 \%$. Febrile Neutropenie Grad 3 und höher sowie Hypertonie traten in der BTH-Gruppe häufiger auf. Hohe Plasmakonzentrationen von VEGF-A vor Therapiestart waren tendenziell mit einem Benefit durch die Kombination mit Bevacizumab assoziiert.

Fazit: Die Kombination von Bevacizumab mit Trastuzumab/Docetaxel führt zu keiner signifikanten Verbesserung des progressionsfreien Überlebens von Patientinnen mit einem metastasierten, HER2-positiven Mammakarzinom. Die Rolle von Plasma-VEGF-A als möglicher Prädiktor für das Therapieansprechen rechtfertigt deren weitere prospektive Evaluation, so die Autoren.

Wolfgang Zimmermann

Gianni L et al. AVEREL: a randomized phase III trial evaluating bevacizumab in combination with docetaxel and trastuzumab as first-line therapy for HER2-positive locally recurrent/ metastatic breast cancer. J Clin Oncol. 2013;31(14):1719-25. 